Bull. Austral. Math. Soc.

VOL. 69 (2004) [191-202]

\title{
ON INVOLUTIVE LIE ALGEBRAS HAVING A CARTAN DECOMPOSITION
}

\section{A.J. Calderón Martín}

We introduce the concept of Cartan decomposition relative to a Cartan subalgebra $H$ in the sense of Y. Billig and A. Pianzola for involutive complex Lie algebras $L$ of arbitrary dimension. If $L$ has such a decomposition and is infinite dimensional and simple, we show it is *-isomorphic to a direct limit of classical finite dimensional simple involutive Lie algebras of the same type $A, B, C$ or $D$.

\section{Preliminaries}

Let $L$ be a complex Lie algebra. An involution on $L$ is a conjugate-linear map, $*: L \rightarrow L\left(x \mapsto x^{*}\right)$, such that $\left(x^{*}\right)^{*}=x$ and $[x, y]^{*}=\left[y^{*}, x^{*}\right]$ for any $x, y \in L$. A Lie algebra furnished with an involution is an involutive Lie algebra. A selfadjoint subset of an involutive algebra is a subset globally invariant by the involution. If $L_{i}(i=1,2)$ are involutive Lie algebras and $f: L_{1} \longrightarrow L_{2}$ is a morphism of Lie algebras, we say that $f$ is a *-morphism whenever $f\left(x^{*}\right)=f(x)^{*}$ for all $x \in L_{1}$. We define the Annihilator of an involutive Lie algebra $L$ as the selfadjoint ideal given by $\operatorname{Ann}(L)=\{x \in L:[x, y]$ $=0$ for all $y \in L\}$. We shall say that $L$ is simple if the product is nonzero and its only ideals are $\{0\}$ and $L$.

Billig and Pianzola introduced in [2] the concept of Cartan subalgebra for Lie algebras $L$ of arbitrary dimension as follows:

Definition 1.1: A subalgebra $H$ of $L$ is called a Cartan subalgebra if

(1) The elements of $H$ act locally ad-nilpotently on $H$.

(2) $H$ is its own normaliser in $L$, that is, $N_{L}(H)=H$.

If $L$ is finite dimensional, then $H$ is nilpotent by Engel's theorem and the classical definition of Cartan subalgebra is recovered.

In the framework of involutive Lie algebras we are interested in selfadjoint Cartan subalgebras of $L$. From here, unless otherwise stated, throughout the paper $H$ shall

Received 1st Apri, 2003

Supported by the PCI of the UCA. 'Teoría de Lie y teoría de Espacios de Banach' and by the PAI of the spanish Junta de Andalucía with project number FQM-298.

Copyright Clearance Centre, Inc. Serial-fee code: 0004-9727/04 \$A2.00+0.00. 
denote a selfadjoint Cartan subalgebra of an involutive complex Lie algebra of arbitrary dimension $L$.

A root of $L$ relative to $H$ is a linear form commuting with the involution

$$
\alpha:(H, *) \rightarrow\left(\mathbb{C},{ }^{-}\right)
$$

that is, $\alpha\left(h^{*}\right)=\overline{\alpha(h)}$ for any $h \in H$, (where - denotes the conjugation operator on $\mathbb{C}$ ), such that there exists $v_{\alpha} \in L, v_{\alpha} \neq 0$ satisfying $\left[h, v_{\alpha}\right]=\alpha(h) v_{\alpha}$ for any $h \in H$. The root space associated to $\alpha$ is the subspace $L_{\alpha}=\left\{v_{\alpha} \in L:\left[h, v_{\alpha}\right]=\alpha(h) v_{\alpha}\right.$ for any $\left.h \in H\right\}$. It is easy to prove that the root space associated to the zero root is contained in the Cartan subalgebra and, by the Jacobi identity, that if $\alpha+\beta$ is a root then $\left[L_{\alpha}, L_{\beta}\right] \subseteq L_{\alpha+\beta}$, and if $\alpha+\beta$ is not a root then $\left[L_{\alpha}, L_{\beta}\right]=0$. Let us also note that $\left(L_{\alpha}\right)^{*}=L_{-\alpha}$. Indeed, for any $h \in H$ and $v_{\alpha} \in L_{\alpha},\left[h, v_{\alpha}\right]^{*}=\left(\alpha(h) v_{\alpha}\right)^{*}=\overline{\alpha(h)} v_{\alpha}^{*}$, and from here $\left[h^{*}, v_{\alpha}^{*}\right]=-\overline{\alpha(h)} v_{\alpha}^{*}=-\alpha\left(h^{*}\right) v_{\alpha}^{*}$, the facts $H^{*}=H$ and $*^{2}=*$ let us conclude easily the assertion. Given a set $S$ of nonzero roots of $L$, we shall denote by $\operatorname{Sp}_{\mathbb{Z}} S$ the set of mappings

$$
\mathrm{Sp}_{\mathbb{Z}} S=\left\{\sum_{i=1}^{n} p_{i} \alpha_{i}: p_{i} \in \mathbb{Z} \text { and } \alpha_{i} \in S\right\} .
$$

DEFINITION 1.2: We shall call that $L$ has a Cartan decomposition relative to $H$ if (1) $L=H \oplus\left(\bigoplus_{\alpha \in \Lambda} L_{\alpha}\right)$, where $\Lambda$ is the set of all nonzero roots of $L$ relative to $H$.

(2) Each $L_{\alpha}, \alpha \in \Lambda$, is finite dimensional.

(3) For any finite set $S \subset \Lambda$ we have $\operatorname{Sp}_{\mathbb{Z}} S \cap \Lambda$ is also finite.

(4) There exists $v_{\alpha} \in L_{\alpha}$ such that $\alpha\left(\left[v_{\alpha}, v_{\alpha}^{*}\right]\right) \in \mathbb{R}^{+}-\{0\}$ for any $\alpha \in \Lambda$.

By using the ideas in $[11,10,16]$ one could characterise infinite dimensional simple involutive Lie algebras over a field $\mathbb{K}$ of characteristic zero, however, we use entirely different methods to describe the complex case. In fact, the introduction of new techniques, such as the connections of roots to construct a direct system of adequate finite dimensional simple involutive Lie algebras, in the study of infinite dimensional Lie algebras is perhaps the most interesting novelty in this paper.

DEFINITION 1.3: Let $(I, \leqslant)$ be a directed set and $\left\{L_{i}\right\}_{i \in I}$ a family of involutive Lie algebras such that for $i \leqslant j$ there exists a *-monomorphism $e_{j i}: L_{i} \longrightarrow L_{j}$ such that $e_{j i} e_{i k}=e_{j k}$ and $e_{i i}=I d$ for all $i, j, k \in I$ with $k \leqslant i \leqslant j$. Then we shall say that $\mathcal{S}:=\left(\left\{L_{i}\right\}_{i \in I},\left\{e_{j i}\right\}_{i \leqslant j}\right)$ is a direct system of involutive Lie algebras.

DEFINITION 1.4: Given $\mathcal{S}$ we define a direct limit, $\underset{\lim }{\longrightarrow} \mathcal{S}$, as a couple $\left(L,\left\{e_{i}\right\}_{i \in I}\right)$ where $L$ is an involutive Lie algebra, $e_{i}: L_{i} \longrightarrow L$ is a *-monomorphism that satisfies $e_{i}=e_{j} e_{j i}$ and $\left(L,\left\{e_{i}\right\}_{i \in I}\right)$ is universal for this property in the sense that if $\left(B,\left\{t_{i}\right\}_{i \in I}\right)$ is another such couple, then there exists a unique $*$-monomorphism $\theta: L \longrightarrow B$ such that $t_{i}=\theta e_{i}, i \in I$. 
As in [3], we can prove that any direct system of involutive Lie algebras $\mathcal{S}$ has a direct limit. It is also clear that $\lim _{\longrightarrow} \mathcal{S}$ is unique up to $*$-isomorphisms.

\section{THE DESCRIPTION THEOREM}

Unless otherwise stated, throughout this section $L$ shall denote an infinite dimensional involutive Lie algebra with zero annihilator having a Cartan decomposition respect to $H$, and $\Lambda$ the set of all nonzero roots.

LEMMA 2.1. The following assertions hold:

(1) $\alpha\left(h_{\alpha}\right) \neq 0$ for any $0 \neq h_{\alpha} \in\left[L_{\alpha}, L_{\alpha}^{*}\right], \alpha \in \Lambda$.

(2) If $\left[L_{\alpha}, L_{\beta}\right]=\left[L_{-\alpha}, L_{\beta}\right]=0$ then $\beta\left(h_{\alpha}\right)=0$ for any $h_{\alpha} \in\left[L_{\alpha}, L_{\alpha}^{*}\right], \alpha, \beta \in \Lambda$.

Proof: 1. Similar to [5, Corollary 1], that is, if $h_{\alpha}=\left[v_{\alpha}, w_{\alpha}^{*}\right]$ with $v_{\alpha}, w_{\alpha}$ $\in L_{\alpha}-\{0\}$ we first observe that for any $\beta \in \Lambda$ the following equation holds

$$
\beta\left(h_{\alpha}\right)=r \alpha\left(h_{\alpha}\right)
$$

with $r \in \mathbb{Q}$, this fact being consequence of $V:=\mathcal{L}\left(L_{\beta+j \alpha}: j \in \mathbb{Z}\right)$, the linear space generated by $\left\{L_{\beta+j \alpha}: j \in \mathbb{Z}\right\}$, is a finite dimensional vector space invariant for $\operatorname{ad}\left(v_{\alpha}\right)$, $\operatorname{ad}\left(w_{\alpha}^{*}\right)$ and $\operatorname{ad}\left(h_{\alpha}\right)=\operatorname{ad}\left(v_{\alpha}\right) \operatorname{ad}\left(w_{\alpha}^{*}\right)-\operatorname{ad}\left(w_{\alpha}^{*}\right) \operatorname{ad}\left(v_{\alpha}\right)$ on which the trace of $\operatorname{ad}\left(h_{\alpha}\right)$ is 0 and so $m \beta\left(h_{\alpha}\right)+k \alpha\left(h_{\alpha}\right)=0$ with $m \neq 0$ and $m, k \in \mathbb{Z}$. Second, if $\alpha\left(h_{\alpha}\right)=0$ then by equation (1), $\beta\left(h_{\alpha}\right)=0$ for all nonzero root $\beta$ and so $\left[h_{\alpha}, L_{\beta}\right]=0$. As $h_{\alpha} \in\left[L_{\alpha}, L_{-\alpha}\right] \subset L_{0}$, we also have $\left[h_{\alpha}, H\right]=0$ and then $\left[h_{\alpha}, L\right]=0$. Hence, $h_{\alpha} \in \operatorname{Ann}(L)$ and so $h_{\alpha}=0$.

2. It is an easy consequence of the Jacobi identity and the fact $L_{\alpha}^{*}=L_{-\alpha}$.

LEMma 2.2. For any $\alpha \in \Lambda$ we have $\operatorname{dim} L_{\alpha}=1$ and $\mathbb{Z} \alpha \cap \Lambda= \pm \alpha$.

Proof: We argue as in [15, Proposition I.6], that is, Lemma 2.1 gives us, for any nonzero elements $v_{\alpha} \in L_{\alpha}, w_{\alpha}^{*} \in L_{\alpha}^{*}$ such that $\left[v_{\alpha}, w_{\alpha}^{*}\right] \neq 0$, that $\alpha\left(\left[v_{\alpha}, w_{\alpha}^{*}\right]\right) \neq 0$ and so the subalgebra $\operatorname{span}_{\mathbb{C}}\left\{v_{\alpha}, w_{\alpha}^{*},\left[v_{\alpha}, w_{\alpha}^{*}\right]\right\}$ is isomorphic to $\mathbf{s l}(2, \mathbb{C})$, we may without loss of generality assume that $\alpha\left(\left[v_{\alpha}, w_{\alpha}^{*}\right]\right)=2$. Condition 3 in Definition 1.2 implies the operators $\operatorname{ad}\left(v_{\alpha}\right)$ and $\operatorname{ad}\left(w_{\alpha}^{*}\right)$ are locally nilpotent on $L$, by using now the same arguments as for $\operatorname{sl}(2, \mathbb{C})$ (see [9, Proposition 2.4.7]) we obtain $L$ is a locally finite $\operatorname{span}_{\mathbb{C}}\left\{v_{\alpha}, w_{\alpha}^{*},\left[v_{\alpha}, w_{\alpha}^{*}\right]\right\}$-module with respect to the adjoint representation. Let us consider the $\operatorname{span}_{\mathbb{C}}\left\{v_{\alpha}, w_{\alpha}^{*},\left[v_{\alpha}, w_{\alpha}^{*}\right]\right\}$-submodule of $L, V:=\mathbb{C} w_{\alpha}^{*}+H+\sum_{n=1}^{\infty} L_{n \alpha}$. As a submodule of a locally finite module, $V$ is also locally finite. Hence the representation theory of $\operatorname{sl}(2, \mathbb{C})$ implies that the set of $h_{\alpha^{-}}$eigenvalues on $V$ is symmetric with

$$
\operatorname{dim} V^{\mu}\left(h_{\alpha}\right)=\operatorname{dim} V^{-\mu}\left(h_{\alpha}\right)
$$

for each $\mu \in \mathbb{C}$. Now $V^{-2}\left(h_{\alpha}\right)=\mathbb{C} w_{\alpha}^{*}$ implies that $\operatorname{dim} V^{2}\left(h_{\alpha}\right)=\operatorname{dim} L_{\alpha}=1$ and furthermore that

$$
\operatorname{dim} V^{2 n}\left(h_{\alpha}\right)=\operatorname{dim} L_{n \alpha}=0
$$


for $n>1$. Since we can replace $\alpha$ by $-\alpha$ in the argument, we have both conclusions of the lemma.

Lemma 2.2 and condition 4 in Definition 1.2 show that given $\alpha \in \Lambda$ there exists a unique nonzero element of $L_{0} \subset H$ of the form

$$
h_{\alpha}=\left[e_{\alpha}, e_{\alpha}^{*}\right]
$$

with $e_{\alpha} \in L_{\alpha}-\{0\}$, and such that $\alpha\left(h_{\alpha}\right)=2$. Let us observe that $e_{\alpha}$ is unique up to a scalar factor of modulus 1. From now on $h_{\alpha}$ shall denote this element.

DEFinition 2.3: A subset $\Lambda_{0}$ of $\Lambda$ is called a root system (relative to $H$ ) if it satisfies the conditions: $\alpha \in \Lambda_{0}$ implies $-\alpha \in \Lambda_{0} ;$ and $\alpha, \beta \in \Lambda_{0}, \alpha+\beta$ $\in \Lambda$ implies $\alpha+\beta \in \Lambda_{0}$. If we define $H_{\Lambda_{0}}$ as $\operatorname{span}_{\mathbb{C}}\left\{h_{\alpha}: \alpha \in \Lambda_{0}\right\}$ and $V_{\Lambda_{0}}=\bigoplus_{\alpha \in \Lambda_{0}} L_{\alpha}$, it is straightforward to verify that $L_{\Lambda_{0}}=H_{\Lambda_{0}} \oplus V_{\Lambda_{0}}$ is an involutive Lie subalgebra of $L$, with Cartan subalgebra $H_{\Lambda_{0}}=H \cap L_{\Lambda_{0}}$, whose roots relative to $H_{\Lambda_{0}}$ are precisely the roots in $\Lambda_{0}$. We shall say that $L_{\Lambda_{0}}$ is the involutive Lie subalgebra associated to the root system $\Lambda_{0}$. Let us observe that if $\Lambda_{0}$ is finite then $L_{\Lambda_{0}}$ is finite dimensional.

Our next goal is to prove the following result.

THEOREM 2.4. Let $L$ be an infinite dimensional simple involutive Lie algebra having a Cartan decomposition respect to $H$. Then there exists a direct system of finite dimensional simple involutive Lie subalgebras $\mathcal{S}:=\left(\left\{L_{i}\right\}_{i \in I},\left\{e_{j i}\right\}_{i \leqslant j}\right)$, with Cartan subalgebras $H_{i}=H \cap L_{i}$ and satisfying

(1) If $i \leqslant j$ then $L_{i}$ is an involutive Lie subalgebra of $L_{j}, e_{j i}$ is the inclusion mapping and each root space of $L_{i}$ relative to $H_{i}$, different to $H_{i}$, is a root space of $L_{j}$.

(2) $\lim _{\longrightarrow} \mathcal{S}=L$.

The arguments we are going to use in the proof of Theorem 2.4 are close to the ones developed in [6, Section IV]. For the convenience of the reader we summarise some of the results in [6, Section IV] with a sketch of the proofs, and some auxiliary lemmas before proving Theorem 2.4.

LEMMA 2.5. Let $L_{\Lambda_{0}}$ be the involutive Lie subalgebra associated to a finite root system $\Lambda_{0}$. Write $\langle\cdot, \cdot\rangle$ the Killing form on $L_{\Lambda_{0}}$. Then the following assertions hold:

(1) $\left\langle h_{\alpha}, h_{\alpha}\right\rangle \neq 0$ for any $\alpha \in \Lambda_{0}$.

(2) $\left\langle h, v_{\alpha}\right\rangle=0$ for any $h \in H_{\Lambda_{0}}$ and $v_{\alpha} \in L_{\alpha}, \alpha \in \Lambda_{0}$.

(3) $\left\langle v_{\alpha}, v_{\beta}\right\rangle=0$ for any $v_{\alpha} \in L_{\alpha}, v_{\beta} \in L_{\beta}, \alpha, \beta \in \Lambda_{0}$ and $\beta \neq-\alpha$.

(4) $\left\langle v_{\alpha}, v_{-\alpha}\right\rangle \neq 0$ for any $0 \neq v_{i \alpha} \in L_{i \alpha}, i \in\{ \pm 1\}$ and $\alpha \in \Lambda_{0}$.

Proof: 1. We have $\left\langle h_{\alpha}, h_{\alpha}\right\rangle=\operatorname{trz}\left(\operatorname{ad}\left(h_{\alpha}\right) \circ \operatorname{ad}\left(h_{\alpha}\right)\right)=\alpha\left(h_{\alpha}\right)^{2}+\sum_{\gamma \in \Lambda_{0}-\{\alpha\}} \gamma\left(h_{\alpha}\right)^{2}$. As in the proof of Lemma 2.1-1 we obtain $\gamma\left(h_{\alpha}\right)=r_{\gamma} \alpha\left(h_{\alpha}\right)$ with $r_{\gamma} \in \mathbb{Q}$, and finally we conclude from $\alpha\left(h_{\alpha}\right)=2$ that $\left\langle h_{\alpha}, h_{\alpha}\right\rangle=4+4 \sum_{\gamma \in \Lambda_{0}-\{\alpha\}} r_{\gamma}^{2} \neq 0$. 
2. Since $\langle\cdot, \cdot\rangle$ is invariant in the sense of $[8$, p. 69], we have

$$
\left\langle h, v_{\alpha}\right\rangle=\frac{1}{2}\left\langle h,\left[h_{\alpha}, v_{\alpha}\right]\right\rangle=\frac{1}{2}\left\langle\left[h, h_{\alpha}\right], v_{\alpha}\right\rangle=0 .
$$

3. It is clear that $\left\langle v_{\alpha}, v_{\beta}\right\rangle=\operatorname{trz}\left(\operatorname{ad}\left(v_{\alpha}\right) \circ \operatorname{ad}\left(v_{\beta}\right)\right)=0$.

4. Since $L_{\alpha}^{*}=L_{-\alpha}$,

$$
\left\langle h_{\alpha}, h_{\alpha}\right\rangle=\left\langle\left[e_{\alpha}, e_{\alpha}^{*}\right], h_{\alpha}\right\rangle=\left\langle e_{\alpha},\left[h_{\alpha}, e_{\alpha}^{*}\right]\right\rangle=-2\left\langle e_{\alpha}, e_{\alpha}^{*}\right\rangle .
$$

By applying 1 . we have $\left\langle e_{\alpha}, e_{\alpha}^{*}\right\rangle \neq 0$. Hence, as $\operatorname{dim} L_{ \pm \alpha}=1$ we conclude $\left\langle v_{\alpha}, v_{-\alpha}\right\rangle \neq 0$.

LEMmA 2.6. Under the hypothesis of Lemma 2.5, if $\left\langle x, L_{\Lambda_{0}}\right\rangle=0$ for some $x$ $\in L_{\Lambda_{0}}$ then $x \in H_{\Lambda_{0}}$.

PROOF: Write $x=h+\sum_{\alpha \in \Lambda_{0}} w_{\alpha} \in L_{\Lambda_{0}}$, with $h \in H_{\Lambda_{0}}$ and $w_{\alpha} \in L_{\alpha}$. Since $\left\langle x, v_{-\alpha}\right\rangle=0$ for any $v_{-\alpha} \in L_{-\alpha}, \alpha \in \Lambda_{0}$, Lemma $2.5-2,3$ shows $\left\langle w_{\alpha}, v_{-\alpha}\right\rangle=0$, and therefore $w_{\alpha}=0$ by Lemma $2.5-4$.

Proposition 2.7. The involutive Lie subalgebra $L_{\Lambda_{0}}$ associated to a finite root system $\Lambda_{0}$ in $L$ is semisimple.

Proof: Let us firstly observe that if we denote by $\left[L_{\Lambda_{0}}, L_{\Lambda_{0}}\right]:=\operatorname{span}_{\mathbb{C}}\{[x, y]: x, y$ $\left.\in L_{\Lambda_{0}}\right\}$, then $\left[L_{\Lambda_{0}}, L_{\Lambda_{0}}\right]=L_{\Lambda_{0}}$ and

$$
\operatorname{Rad}\left(L_{\Lambda_{0}}\right) \subset H_{\Lambda_{0}}
$$

Indeed, if $x \in L_{\Lambda_{0}}$ then

$$
x=\sum_{\alpha \in \Lambda_{0}} \lambda_{\alpha} h_{\alpha}+\sum_{\alpha \in \Lambda_{0}} v_{\alpha}=\sum_{\alpha \in \Lambda_{0}} \lambda_{\alpha}\left[e_{\alpha}, e_{\alpha}^{*}\right]+\frac{1}{2} \sum_{\alpha \in \Lambda_{0}}\left[h_{\alpha}, v_{\alpha}\right] \in\left[L_{\Lambda_{0}}, L_{\Lambda_{0}}\right]
$$

and so $\left[L_{\Lambda_{0}}, L_{\Lambda_{0}}\right]=L_{\Lambda_{0}}$. Since the radical of a finite dimensional Lie algebra $L^{\prime}$ is characterised as the ideal $\operatorname{Rad}\left(L^{\prime}\right)=\left\{x \in L^{\prime}:\left\langle x,\left[L^{\prime}, L^{\prime}\right]\right\rangle=0\right\}$, where $\langle\cdot, \cdot\rangle$ denotes the Killing form (see [8, p. 73]), the fact $\left[L_{\Lambda_{0}}, L_{\Lambda_{0}}\right]=L_{\Lambda_{0}}$ and Lemma $2.6 \operatorname{show} \operatorname{Rad}\left(L_{\Lambda_{0}}\right)$ $\subset H_{\Lambda_{0}}$.

Secondly, we assert that

$$
\operatorname{Rad}\left(L_{\Lambda_{0}}\right)=\operatorname{Ann}\left(L_{\Lambda_{0}}\right) .
$$

Indeed, $\operatorname{Ann}\left(L_{\Lambda_{0}}\right)$ is a solvable ideal and therefore is included in $\operatorname{Rad}\left(L_{\Lambda_{0}}\right)$. If $h$ $\in \operatorname{Rad}\left(L_{\Lambda_{0}}\right) \subset H_{\Lambda_{0}}$ we have, by the character of ideal of $\operatorname{Rad}\left(L_{\Lambda_{0}}\right),\left[h, v_{\alpha}\right] \in \operatorname{Rad}\left(L_{\Lambda_{0}}\right)$ $\subset H_{\Lambda_{0}}$ for any $0 \neq v_{\alpha} \in L_{\alpha}$ and $\alpha \in \Lambda_{0}$, therefore $\alpha(h)=0$. Hence, we have for any $x \in L_{\Lambda_{0}}$,

$$
[h, x]=\left[h, \sum_{\alpha \in \Lambda_{0}} \lambda_{\alpha} h_{\alpha}+\sum_{\alpha \in \Lambda_{0}} v_{\alpha}\right]=\left[h, \sum_{\alpha \in \Lambda_{0}} \lambda_{\alpha} h_{\alpha}\right]+\sum_{\alpha \in \Lambda_{0}}\left[h, v_{\alpha}\right]=0
$$


and so $h \in \operatorname{Ann}\left(L_{\Lambda_{0}}\right)$.

Finally, as by Levi's theorem, $([8$, p. 91$])$,

$$
L_{\Lambda_{0}}=\operatorname{Rad}\left(L_{\Lambda_{0}}\right) \oplus T_{\Lambda_{0}}
$$

with $T_{\Lambda_{0}}$ a semisimple subalgebra of $L_{\Lambda_{0}}$, we have

$$
L_{\Lambda_{0}}=\left[L_{\Lambda_{0}}, L_{\Lambda_{0}}\right]=\left[\operatorname{Ann}\left(L_{\Lambda_{0}}\right) \oplus T_{\Lambda_{0}}, \operatorname{Ann}\left(L_{\Lambda_{0}}\right) \oplus T_{\Lambda_{0}}\right] \subset\left[T_{\Lambda_{0}}, T_{\Lambda_{0}}\right] \subset T_{\Lambda_{0}}
$$

and so $L_{\Lambda_{0}}=T_{\Lambda_{0}}$, the proof is complete.

We shall say that a finite set of nonzero roots $\left\{\alpha_{i}\right\}$ of $L$ is linearly independent if the set $\left\{h_{\alpha_{i}}\right\}$ is linearly independent. We also recall that an $L^{*}$-algebra is defined, (see $[13,14,7])$, as a complex involutive Hilbert-Lie algebra for which the inner product $(\cdot \mid \cdot)$ satisfies the $H^{*}$-identities

$$
([x, y] \mid z)=\left(y \mid\left[x^{*}, z\right]\right)=\left(x \mid\left[z, y^{*}\right]\right) .
$$

J.R. Schue introduced for any non zero root $\alpha$ of a semisimple $L^{*}$-algebra $L^{\prime}$ with inner product $(\cdot \mid \cdot)$ and with a Cartan decomposition $L^{\prime}=\overline{H^{\prime}+\sum L_{\alpha}^{\prime}}$, the elements $0 \neq h_{\alpha}^{\prime} \in\left[L_{\alpha}^{\prime}, L_{-\alpha}^{\prime}\right]$ satisfying $\alpha\left(h^{\prime}\right)=\left(h^{\prime} \mid h_{\alpha}^{\prime}\right)$ for any $h^{\prime} \in H^{\prime}$ (see [1, pp. 513-514] or [13, pp. 71-72]). It is well known, (see [4, Proof of Proposition 3.1] or the ideas in [12]), that any complex finite dimensional semisimple Lie algebra with a Cartan decomposition $L=H+\sum L_{\alpha}$ and with the expression $L=\bigoplus_{j=1}^{m} L_{j}$, where $L_{j}$ are simple Lie algebras, admits an, essentially unique, involution $*^{\prime}$ and inner product $(\cdot \mid \cdot)$ that make $L$ an $L^{*}$-algebra admitting the same Cartan decomposition $L=H+\sum L_{\alpha}$, and such that $\left(L_{i} \mid L_{j}\right)=0$ for $i \neq j$. Since we can see a finite dimensional semisimple involutive Lie algebra having a Cartan decomposition $L=H+\sum L_{\alpha}$ as a Lie algebra which also admits the Cartan decomposition (in the classical sense) $L=H+\sum L_{\alpha}$, the above considerations imply in this framework $h_{\alpha}^{\prime}=k h_{\alpha}$ with $0 \neq k \in \mathbb{C}-\{0\}$, and joint with [1, Lemma 1] and $[1$, Corollary 2$]$ give us the following two results:

LEMMA 2.8. Let $L$ be a finite dimensional semisimple involutive Lie algebra having a Cartan decomposition relative to $H$. Write $L$ as $L=\bigoplus_{j=1}^{m} L_{j}$ where $L_{j}$ are simple Lie algebras. If $\alpha$ is a nonzero root relative to $H$, then $L_{\alpha}$ belongs precisely to one $L_{j}$. If we denote by

$$
\Lambda_{j}=\left\{\alpha: L_{\alpha} \subseteq L_{j}\right\},
$$

then $\operatorname{span}_{\mathbb{C}}\left\{\left[v_{\alpha}, v_{-\alpha}\right]: \alpha \in \Lambda_{j}\right\}$ is a Cartan subalgebra $H_{j}$ of $L_{j}$ and the restrictions to $H_{j}$ of the $\alpha \in \Lambda_{j}$ are precisely the roots of $L_{j}$.

Corollary 2.9. Let $L$ be as in Lemma 2.8. Let us suppose $\left\{\alpha_{1,}, \ldots, \alpha_{n}\right\}$ is a linearly independent set of nonzero roots of $L$. If there exists a root $\gamma$ of $L$ such that $\gamma=\sum_{i=1}^{n} c_{i} \alpha_{i} \quad c_{i} \neq 0$, then all $\alpha_{i}$ and $\gamma$ are roots of the same simple component $L_{j}$. 
LEMMA 2.10. Let $L$ be as in Lemma 2.8. If $\alpha$ and $\beta$ are two nonzero roots such that $\alpha \neq \pm \beta$ then $\alpha$ and $\beta$ are linearly independent.

Proof: Suppose $\alpha$ and $\beta$ are not linearly independent, then $h_{\alpha}=c h_{\beta}$ with $0 \neq c$ $\in \mathbb{C}$. Let consider $L$ as an $L^{*}$-algebra with inner product $(\cdot \mid \cdot)$. By the above observation, there exist non zero elements $h_{\alpha}^{\prime}, h_{\beta}^{\prime} \in H$ such that $\alpha(h)=\left(h \mid h_{\alpha}^{\prime}\right)$ and $\beta(h)=\left(h \mid h_{\beta}^{\prime}\right)$ for any $h \in H$ and $h_{\alpha}^{\prime}=k_{\alpha} h_{\alpha}, h_{\beta}^{\prime}=k_{\beta} h_{\beta}$ with $k_{\alpha}, k_{\beta} \in \mathbb{C}-\{0\}$. Hence,

$$
\alpha(h)=\left(h \mid k_{\alpha} h_{\alpha}\right)=\left(h \mid k_{\alpha} c h_{\beta}\right)=\left(h \mid k_{\alpha} c k_{\beta}^{-1} h_{\beta}^{\prime}\right)=\overline{k_{\alpha} c k_{\beta}^{-1}} \beta(h)
$$

for any $h \in H$. From the theory of finite dimensional split semisimple Lie algebras, this is only possible if $\alpha= \pm \beta$.

DEFINITION 2.11: Let $\alpha$ and $\beta$ be two nonzero roots of an involutive Lie algebra with zero annihilator, we shall say that $\alpha$ and $\beta$ are connected if there exist $\alpha_{1}, \ldots, \alpha_{n} \in \Lambda$ such that

$$
\left\{\alpha_{1}, \alpha_{1}+\alpha_{2}, \alpha_{1}+\alpha_{2}+\alpha_{3}, \ldots, \alpha_{1}+\cdots+\alpha_{n-1}+\alpha_{n}\right\}
$$

is a family of nonzero roots, $\alpha_{1}$ is a fixed element of $\{\alpha,-\alpha\}$ and $\alpha_{1}+\cdots+\alpha_{n-1}+\alpha_{n}=\beta$. We shall also say that $\left\{\alpha_{1}, \ldots, \alpha_{n}\right\}$ is a connection from $\alpha$ to $\beta$.

It is clear that

$$
\alpha_{p} \neq \pm \sum_{i=1}^{p-1} \alpha_{i}, \quad p=2, . ., n
$$

We denote by

$$
\Lambda_{\alpha}:=\{\beta \in \Lambda: \alpha \text { and } \beta \text { are connected }\}
$$

Let us observe that $\{\alpha\}$ is a connection from $\alpha$ to itself and therefore $\alpha \in \Lambda_{\alpha}$.

LEMMA 2.12. Under the hypothesis of Lemma 2.8, and if in addition $\alpha$ and $\beta$ are two connected nonzero roots, then $L_{\alpha}$ and $L_{\beta}$ belong to the same simple Lie algebra $L_{j}$.

Proof: We have $\alpha_{1}, \ldots, \alpha_{n} \in \Lambda$ such that

$$
\left\{\alpha_{1}, \alpha_{1}+\alpha_{2}, \ldots, \alpha_{1}+\cdots+\alpha_{n-1}+\alpha_{n}\right\}
$$

are nonzero roots, $\alpha_{1}$ is a fixed element of $\{\alpha,-\alpha\}$ and $\alpha_{1}+\cdots+\alpha_{n-1}+\alpha_{n}=\beta$. If we consider $\alpha_{1}, \alpha_{2}$, and $\alpha_{1}+\alpha_{2}$, by (3) $\alpha_{2} \neq \pm \alpha_{1}$, then Lemma 2.10 gives us that $\alpha_{1}$ and $\alpha_{2}$ are linearly independent and finally Corollary 2.9 let us conclude $L_{\alpha_{1}}, L_{\alpha_{2}}$ and $L_{\alpha_{1}+\alpha_{2}}$ belong to the same simple Lie algebra $L_{j}$. The same argument with $\alpha_{1}+\alpha_{2}, \alpha_{3}$ and $\alpha_{1}+\alpha_{2}+\alpha_{3}$ gives us $L_{\alpha_{3}}, L_{\alpha_{1}+\alpha_{2}+\alpha_{3}} \subset L_{j}$. Following this process we finally obtain $L_{\alpha}, L_{\beta} \subset L_{j}$.

Proposition 2.13. Let $L$ be an infinite dimensional simple involutive Lie algebra having a Cartan decomposition respect to $H$, and let $\alpha$ be a nonzero root. Then the following assertions hold: 
(1) $\Lambda_{\alpha}$ is a root system.

(2) There exists $\beta \in \Lambda_{\alpha}$ such that $\beta \neq \pm \alpha$.

(3) If $\gamma$ is a nonzero root such that $\gamma \notin \Lambda_{\alpha}$, then $\left[L_{\beta}, L_{\gamma}\right]=0$ and $\gamma\left(h_{\beta}\right)=0$ for any $\beta \in \Lambda_{\alpha}$.

PROOF: 1. If $\beta \in \Lambda_{\alpha}$ then there exists a connection $\left\{\alpha_{1}, \ldots, \alpha_{n}\right\}$ from $\alpha$ to $\beta$. It is easy to check that $\left\{-\alpha_{1}, \ldots,-\alpha_{n}\right\}$ is a connection from $\alpha$ to $-\beta$ and therefore $-\beta \in \Lambda_{\alpha}$. If $\beta, \gamma \in \Lambda_{\alpha}$ and $\beta+\gamma \in \Lambda$, then there exists a connection $\left\{\alpha_{1}, \ldots, \alpha_{n}\right\}$ from $\alpha$ to $\beta$. Hence, $\left\{\alpha_{1}, \ldots, \alpha_{n}, \gamma\right\}$ is a connection from $\alpha$ to $\beta+\gamma$ and so $\beta+\gamma \in \Lambda_{\alpha}$.

2. Firstly, let us observe that there exists $\gamma \in \Lambda, \gamma \neq \pm \alpha$ such that either $\left[L_{\alpha}, L_{\gamma}\right]$ $\neq 0$ or $\left[L_{-\alpha}, L_{\gamma}\right] \neq 0$. Indeed, if we suppose $\left[L_{\alpha}, L_{\gamma}\right]=\left[L_{-\alpha}, L_{\gamma}\right]=0$ for any $\gamma \in \Lambda$, $\gamma \neq \pm \alpha$, as $L_{-\alpha}=L_{\alpha}^{*}$ then by Lemma 2.1-2 we have $\gamma\left(h_{\alpha}\right)=0$ for any $\gamma \in \Lambda, \gamma \neq \pm \alpha$. Let us consider

$$
I:=\mathbb{C} h_{\alpha} \oplus L_{\alpha} \oplus L_{-\alpha} .
$$

By the above, it is easy to prove that $[I, L] \subset I$, therefore $I$ is a nonzero finite dimensional ideal of an infinite dimensional simple involutive Lie algebra $L$, a contradiction. Hence, there exists a nonzero root $\gamma \neq \pm \alpha$ such that either $\left[L_{\alpha}, L_{\gamma}\right] \neq 0$ or $\left[L_{-\alpha}, L_{\gamma}\right] \neq 0$. In the first case, $\{\alpha, \gamma\}$ is a connection from $\alpha$ to $\beta:=\alpha+\gamma$, therefore $\beta \in \Lambda_{\alpha}$ and $\beta \neq \pm \alpha$. In the second case we argue similarly.

3. Let us suppose there exists $\beta \in \Lambda_{\alpha}$ such that $\left[L_{\beta}, L_{\gamma}\right] \neq 0$. If $\left\{\alpha_{1}, \ldots, \alpha_{n}\right\}$ is a connection from $\alpha$ to $\beta$, we have $\left\{\alpha_{1}, \ldots, \alpha_{n}, \gamma\right\}$ is a connection from $\alpha$ to $\beta+\gamma$. Since $\Lambda_{\alpha}$ is a root system then $\gamma \in \Lambda_{\alpha}$, a contradiction. Therefore $\left[L_{\beta}, L_{\gamma}\right]=0$ for any $\beta \in \Lambda_{\alpha}$ and $\gamma \notin \Lambda_{\alpha}$. As $-\beta \in \Lambda_{\alpha}$ for any $\beta \in \Lambda_{\alpha}$, we also have $\left[L_{-\beta}, L_{\gamma}\right]=0$. Finally, by Lemma 2.1-2 we conclude $\gamma\left(h_{\beta}\right)=0$.

Proposition 2.14. Let $L$ be an infinite dimensional simple involutive Lie algebra having a Cartan decomposition respect to $H$. Then there exists a connection from $\alpha$ to $\beta$ for any $\alpha, \beta \in \Lambda$.

Proof: Let consider the root system $\Lambda_{\alpha}$ and the involutive Lie subalgebra associated

$$
L_{\Lambda_{\alpha}}=H_{\Lambda_{\alpha}} \oplus V_{\Lambda_{\alpha}} .
$$

We assert that $L_{\Lambda_{\alpha}}$ is a nonzero ideal of $L$. Indeed, by Proposition 2.13-3 we have $\left[L_{\beta}, L_{\gamma}\right]=0$ and $\left[h_{\beta}, L_{\gamma}\right]=0$ for any $\beta \in \Lambda_{\alpha}$ and $\gamma \notin \Lambda_{\alpha}$. Hence,

$$
\left[L_{\Lambda_{\alpha}}, L\right]=\left[\sum_{\beta \in \Lambda_{\alpha}} \mathbb{C} h_{\beta}+\sum_{\beta \in \Lambda_{\alpha}} L_{\beta}, H+\left(\sum_{\gamma \in \Lambda_{\alpha}} L_{\gamma}\right)+\left(\sum_{\gamma \notin \Lambda_{\alpha}} L_{\gamma}\right)\right] \subset L_{\Lambda_{\alpha}} .
$$

The simplicity of $L$ implies $L_{\Lambda_{\alpha}}=L$ and therefore $\Lambda_{\alpha}=\Lambda$.

Corollary 2.15. Let $L$ be as in Proposition 2.14. Then, for a fixed $\alpha_{0} \in \Lambda$, we have

$$
L=\underset{\mathbb{C}}{\operatorname{span}}\left\{h_{\beta}: \beta \in \Lambda_{\alpha_{0}}\right\}+\sum_{\beta \in \Lambda_{\mathbf{a}_{0}}} L_{\beta}
$$


DEFINITION 2.16: From now on, we shall consider the classical finite dimensional simple Lie algebras (of types $A, B, C$, or $D$ ) endowed with the standard involution given by $\left(a_{i j}\right)^{*}=\left(\overline{a_{j i}}\right)$. These algebras become involutive Lie algebras with the standard involution and will be called classical finite dimensional simple involutive Lie algebras.

Given a classical finite dimensional simple involutive Lie algebra $L$ of a fixed type $A, B, C$ or $D$, we shall give the name canonical Cartan subalgebra of $L$ to the one described in [8, Chapter IV, 6] for each type.

Proof of Theorem 2.4: 1 . Let $S$ be a non empty finite subset of $\Lambda$, from condition 3 in Definition $1.2, \mathrm{Sp}_{\mathbb{Z}} S \cap \Lambda$ is a finite root system and then we can consider the finite dimensional involutive Lie subalgebra associated $L_{\left(\mathrm{Sp}_{\mathbb{Z}} S \cap \Lambda\right)}$, that we shall denote by $L_{S}:=L_{\left(\mathrm{Sp}_{\mathbb{Z}} S \cap \Lambda\right)}$. By Proposition $2.7, L_{S}$ is semisimple. It is well known from the theory of finite dimensional semisimple Lie algebras that $L_{S}$ can be written

$$
L_{S}=\bigoplus_{i=1}^{n_{S}} L_{S_{i}}
$$

with $L_{S_{i}}, i=1, \ldots, n_{S}$, finite dimensional simple Lie algebras. By Lemma 2.12, we conclude that for any nonzero root $\alpha$ of $L_{S}$ respect to $H \cap L_{S}, L_{ \pm \alpha}$ belong precisely to one $L_{S_{i}}$ and so any $L_{S_{i}}$ is an involutive Lie algebra. Hence, we can consider the family of finite dimensional simple involutive Lie subalgebras of $L$,

$$
\left\{L_{S_{i}}\right\}_{S \in \mathcal{F}, i \in\left\{1, \ldots, n_{S}\right\}}
$$

where $\mathcal{F}$ denotes the family of all non empty finite subset of $\Lambda$. We wish to prove that

$$
\mathcal{S}:=\left(\left\{L_{S_{i}}\right\}_{S \in \mathcal{F}, i \in\left\{1, \ldots, n_{S}\right\}},\left\{i_{S_{i}, T_{j}}\right\}\right),
$$

where $\left\{i_{S_{i}, T_{j}}\right\}$ are the inclusion mappings is the required direct system. We assert that given

$$
L_{S_{i}}, L_{T_{j}} \in\left\{L_{S_{i}}\right\}_{S \in \mathcal{F}, i \in\left\{1, \ldots, n_{s}\right\}}
$$

there exists

$$
L_{Q_{i_{0}}} \in\left\{L_{S_{i}}\right\}_{S \in \mathcal{F}, i \in\left\{1, \ldots, n_{S}\right\}}
$$

such that $L_{S_{i}}, L_{T_{j}} \subset L_{Q_{i_{0}}}$. Indeed, let us fix $\alpha_{0} \in S_{i}$. By Proposition 2.14, for any $\beta \in S_{i} \cup T_{j}$ there exists a connection from $\alpha_{0}$ to $\beta$, which we denote by $C_{\alpha_{0}, \beta}$. We have that $Q:=\bigcup_{\beta \in S_{i} \cup T_{j}} C_{\alpha_{0}, \beta}$ is a finite set of $\Lambda$ and therefore we can consider the finite dimensional semisimple involutive Lie subalgebra associated $L_{Q}$. Write $L_{Q}=\bigoplus_{i=1}^{n_{Q}} L_{Q_{i}}, L_{Q_{i}}$ being simple subalgebras of $L_{Q}$. By Lemma 2.8, there exists $L_{Q_{i_{0}}}$ such that ${ }_{\alpha_{0}}^{i=1} \subset L_{Q_{i_{0}}}$. Finally, by Lemma $2.12, L_{S_{i}}, L_{T_{j}} \subset L_{Q_{i_{0}}}$. Therefore, $\mathcal{S}$ is a direct system with the inclusion which clearly satisfies assertion 1 . of the theorem.

2. Let us denote $\lim _{\longrightarrow} \mathcal{S}=\left(L^{\prime},\left\{e_{j}\right\}_{j}\right)$. As $\left(L,\left\{i_{j}\right\}_{j}\right)$, where $i_{j}$ denotes the inclusion mapping, satisfies the conditions of the direct limit for $\mathcal{S}$, the universal property 
of the direct limits shows the existence of a unique *-monomorphism $\Phi: L^{\prime} \rightarrow L$ such that $\Phi \circ e_{j}=i_{j}$. Since $L^{\prime}=\bigcup_{j} e_{j}\left(L_{j}\right)$, (see for instance [3]), we have $\Phi\left(L^{\prime}\right)$ $=\Phi\left(\bigcup_{j} e_{j}\left(L_{j}\right)\right)=\bigcup_{j} L_{j}$, and therefore $\Phi$ is a $*$-isomorphism from $L^{\prime}$ onto $\bigcup_{j} L_{j}$. Finally, we assert that $L=\bigcup_{j} L_{j}$. Indeed, if $x \in L$, by Proposition 2.14 and Corollary 2.15, $x=\sum_{i=1}^{n} \lambda_{\alpha_{i}} h_{\alpha_{i}}+\sum_{j=1}^{m} v_{\gamma_{j}}$ with $\alpha_{i}, \gamma_{j} \in \Lambda, v_{\gamma_{j}} \in L_{\gamma_{j}}$ and $\lambda_{\alpha_{i}} \in \mathbb{C}$. Consider $T=\left\{\alpha_{i}: i=1, \ldots, n\right\} \cup\left\{\gamma_{j}: j=1, \ldots, m\right\} \subset \Lambda$ and, following the above notation, $T^{\prime}=\bigcup_{\beta \in T} C_{\delta_{0}, \beta}, \delta_{0}$ being a fixed element of $T$. We have $T^{\prime}$ is a finite set of $\Lambda$ that gives us the semisimple finite dimensional involutive Lie algebra associated $L_{T^{\prime}}$. Write $L_{T^{\prime}}=\bigoplus_{i=1}^{r} L_{T_{i}^{\prime}}$, where $L_{T_{i}^{\prime}}, i=1, \ldots, r$ are simple finite dimensional involutive Lie algebras. As $\mathcal{S}$ is a direct system for the inclusion then there exists a finite dimensional simple involutive Lie subalgebra $L_{P_{0}}$ such that $\bigcup_{i=1}^{r} L_{T_{i}^{\prime}} \subseteq L_{P_{0}}$ and therefore $x \in L_{P_{0}}$. The proof of 2 . is complete.

THEOREM 2.17. Let $L$ be an infinite dimensional simple involutive Lie algebra having a Cartan decomposition respect to $H$. Then $L$ is *-isomorphic to a direct limit of classical finite dimensional simple involutive Lie algebras of the same type $A, B, C$ or $D$.

Proof: Let us consider the direct system of finite dimensional simple involutive Lie algebras $\mathcal{S}$ given in Theorem 2.4. We can suppose all of the $L_{i}$ are isomorphic to classical simple Lie algebras of a same type $A, B, C$ or $D$. Indeed, the infinite dimensional character of $L$ let us remove the exceptional Lie algebras of $\mathcal{S}$, and secondly that (i) each $L_{i}$ is contained in one isomorphic to one of type $A$ or else (ii) there exists $L_{i_{0}}$ such that $L_{i} \supset L_{i_{0}}$ implies that $L_{i}$ is isomorphic to one of type $B, C$ or $D$. In each of the two cases is possible to define a subsystem satisfying assertions 1 . and 2. of Theorem 2.4.

If all of the $L_{i}$ are isomorphic to classical simple Lie algebras of type $A$ and we denote by $\phi_{i}: L_{i} \rightarrow A_{i}$ such isomorphisms, we assert that if consider $A_{i}$ as an involutive Lie algebra with its standard involution, then there exists a $*$-isomorphism $\xi_{i}$ from $L_{i}$ onto $A_{i}$. Indeed, $\phi_{i}$ induces on $A_{i}$ a unique Cartan decomposition $A_{i}=H^{\prime} \oplus\left(\underset{\alpha^{\prime} \in \Lambda_{i}^{\prime}}{\bigoplus}\left(A_{i}\right)_{\alpha^{\prime}}\right)$ and involution $*^{\prime}$ that make $\phi_{i}$ a $*$-isomorphism. On the other hand, if we consider $A_{i}$ with its canonical Cartan decomposition given in [8, p. 136-137], $A_{i}=H^{\prime \prime} \oplus\left(\underset{\alpha^{\prime \prime} \in \Lambda_{i}^{\prime \prime}}{\bigoplus}\left(A_{i}\right)_{\alpha^{\prime \prime}}\right)$, it is well known from the theory of finite dimensional Lie algebras, see [8, Chapter IX, Theorem 3], that there exists an automorphism $\mu_{i}: A_{i} \rightarrow A_{i}$ satisfying $\mu_{i}\left(H^{\prime}\right)=H^{\prime \prime}$. As a consequence, we can express the roots $\alpha^{\prime \prime}$ as $\alpha^{\prime \prime}\left(h^{\prime \prime}\right)=\alpha^{\prime}\left(\mu_{i}^{-1}\left(h^{\prime \prime}\right)\right)$ for a certain root $\alpha^{\prime}$. This gives us a a bijection $\alpha^{\prime} \rightarrow \alpha^{\prime \prime}$ satisfying that the Cartan matrices associated to a fixed simple system of roots $\left(\alpha_{1}^{\prime}, \ldots, \alpha_{n}^{\prime}\right),\left(2\left\langle\alpha_{i}^{\prime}, \alpha_{j}^{\prime}\right\rangle /\left\langle\alpha_{i}^{\prime}, \alpha_{i}^{\prime}\right\rangle\right)$ and the one associated to $\left(\alpha_{1}^{\prime \prime}, \ldots, \alpha_{n}^{\prime \prime}\right)$ are identical. Let $e_{\alpha_{i}^{\prime}},\left(e_{\alpha_{i}^{\prime}}\right)^{*^{\prime}}, h_{\alpha_{i}^{\prime}}$ as in $(2)$, the canonical generators for 
$A_{i}$ associated to $\left(\alpha_{1}^{\prime}, \ldots, \alpha_{n}^{\prime}\right)$, and $E_{p q} \in L_{\alpha_{i}^{\prime \prime}}, E_{q p} \in L_{-\alpha_{i}^{\prime \prime}}, E_{p p}-E_{q q} \in H^{\prime \prime}$, (where $E_{r s}$ denotes the elemental matrix), the canonical generators for $A_{i}$ associated to $\left(\alpha_{1}^{\prime \prime}, \ldots, \alpha_{n}^{\prime \prime}\right)$ (see [8, p. 136-137]). By applying the Isomorphism Theorem, [8, Theorem 2 on p. 127], there exists a unique automorphism $\eta_{i}$ of $A_{i}$ mapping $e_{\alpha_{i}^{\prime}}$ on $E_{p q},\left(e_{\alpha_{i}^{\prime}}\right)^{*^{\prime}}$ on $E_{q p}$ and $h_{\alpha_{i}^{\prime}}$ on $E_{p p}-E_{q q}$. Moreover, as $\left\{e_{\alpha_{i}^{\prime}},\left(e_{\alpha_{i}^{\prime}} *^{*^{\prime}}, h_{\alpha_{i}^{\prime}}\right\}\right.$ generates $A_{i}$, ([8, Property XVIII on p. 123]), we can assert $\eta_{i}$ is a $*$-automorphism from $\left(A_{i}, *^{\prime}\right)$ onto $\left(A_{i}, \tau\right), \tau$ being the standard involution $\left(a_{i, j}\right)^{\tau}:=\left(\overline{a_{j, i}}\right)$. Finally, we have $\xi_{i}:=\eta_{i} \circ \phi_{i}$ is $*$-isomorphism from $L_{i}$ onto the classical simple involutive Lie algebra $A_{i}$ as we wished to prove.

If all of the $L_{i}$ are isomorphic to classical Lie algebras $X_{i}$ of a same type $B, C$ or $D$, we argue as in the previous case to find a $*$-isomorphism $\xi_{i}$ from $L_{i}$ onto the classical simple involutive Lie algebra $X_{i}$.

From now on $X$ denotes a classical simple involutive Lie algebra of a fixed type $X=A, B, C$ or $D$. For any couple $i, j \in I$ with $i \leqslant j$, let $e_{j i}$ be the inclusion mapping and $f_{j i}$ the unique $*$-monomorphism making commutative the following diagram

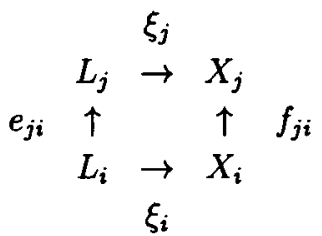

It is clear that

$$
\mathcal{S}^{\sharp}=\left(\left\{X_{i}\right\}_{i \in I},\left\{f_{j i}\right\}_{i, j \in I, i \leqslant j}\right)
$$

is a direct system of classical finite dimensional simple involutive Lie algebras of a same type $X$. Finally, since for any $i, j \in I$ with $i \leqslant j$, we have the $*$-isomorphisms $\xi_{i}$ : $L_{i} \rightarrow X_{i}, \xi_{j}: L_{j} \rightarrow X_{j}$ and the commutativity of the diagrams (4) we conclude $\underline{\longrightarrow} \mathcal{S}$ is *-isomorphic to $\lim _{\longrightarrow} \mathcal{S}^{\sharp}$ and the proof is complete.

\section{REFERENCES}

[1] V.K. Balachandran, 'Simple Systems of Roots in $L^{*}$-algebras', Trans. Amer. Math. Soc. 130 (1968), 513-524.

[2] Y. Billig and A. Pianzola, 'On Cartan subalgebras', J. Algebra 171 (1995), 397-412.

[3] A.J. Calderón and C. Martín, 'Direct limits of $L^{*}$-triples', Algebras Groups Geom. 18 (2001), 223-232.

[4] A.J. Calderón and C. Martín, 'Hilbert space methods in the theory of Lie triple systems', in Recent Progress in Functional Analysis, (K.D. Bierstedt, J. Bonet, M. Maestre and J. Schmets, Editors), North-Holland Math. Studies 189 (North-Holland Publishing Co., Amsterdam, 2001), pp. 309-319.

[5] A.J. Calderón and M. Forero, 'Roots and roots spaces of compact Lie algebras', Irish Math. Soc. Bull. 49 (2002), 15-22. 
[6] A.J. Calderón and M. Forero, 'On infinite dimensional Lie algebras having a Cartan decomposition', (preprint, Universidad de Cádiz).

[7] J.A. Cuenca, A. García and C. Martín, 'Structure theory for $L^{*}$-algebras', Math. Proc. Cambridge Philos. Soc. 107 (1990), 361-365.

[8] N. Jacobson, Lie algebras (Dover Publications, Inc., New York, N.Y., 1979).

[9] R. Moody and A. Pianzola, Lie algebras with triangular decomposition, Canad. Math. Soc. Series of Monographs and Advanced Texts (J. Wiley \& Sons, New York, 1995).

[10] K.-H. Neeb, 'Locally finite Lie algebras with unitary highest weight representations', Manuscripta Math. 104 (2001), 359-381.

[11] K.-H. Neeb and N. Stumme, 'The classification of locally finite split simple Lie algebras', J. Reine Angew. Math. 533 (2001), 25-53.

[12] E. Neher, 'Cartan-involutionen von halbeinfachen rellen Jordan tripelsystemen', Math. Z. 169 (1979), 271-292.

[13] J.R. Schue, 'Hilbert Space methods in the theory of Lie algebras', Trans. Amer. Math. Soc. 95 (1960), 69-80.

[14] J.R. Schue, 'Cartan decompositions for $L^{*}$-algebras', Trans. Amer. Math. Soc. 98 (1961), 334-349.

[15] N. Stumme, 'The structure of locallyfinite split Lie algebras', J. Algebra 220 (1999), 664-693.

[16] N. Stumme, 'Automorphisms and conjugancy of compact real forms of the classical infinite-dimensional matrix Lie algebras', Forum Math. 13 (2001), 817-851.

Departamento de Matemáticas

Universidad de Cádiz

11510 Puerto Real

Cádiz

e-mail: ajesus.calderon@uca.es 